\title{
Towards a muon radiography of the Puy de Dôme
}

\author{
C. Cârloganu ${ }^{1}$, V. Niess ${ }^{1}$, S. Béné ${ }^{1}$, E. Busato ${ }^{1}$, P. Dupieux ${ }^{1}$, F. Fehr ${ }^{1}$, P. Gay ${ }^{1}$, D. Miallier ${ }^{1}$, B. Vulpescu ${ }^{1}$, P. Boivin ${ }^{2,3,4}$, \\ C. Combaret ${ }^{5}$, P. Labazuy ${ }^{2,3,4}$, I. Laktineh ${ }^{5}$, J.-F. Lénat ${ }^{2,3,4}$, L. Mirabito ${ }^{5}$, and A. Portal ${ }^{2,3,4}$ \\ ${ }^{1}$ Clermont Université, Université Blaise Pascal, CNRS/IN2P3, Laboratoire de Physique Corpusculaire, BP 10448, \\ 63000 Clermont-Ferrand, France \\ ${ }^{2}$ Clermont Université, Université Blaise Pascal, Observatoire de Physique du Globe de Clermont-Ferrand, \\ Laboratoire Magmas et Volcans, BP 10118, 63000 Clermont-Ferrand, France \\ ${ }^{3}$ CNRS - UMR6524, LMV, 63038 Clermont-Ferrand, France \\ ${ }^{4}$ IRD, R 163, LMV, 63038 Clermont-Ferrand, France \\ ${ }^{5}$ Université de Lyon, Université Lyon 1, CNRS/IN2P3, IPNL, 1 Rue E. Fermi, 69622 Villeurbanne Cedex, France \\ Correspondence to: V. Niess (niess@in2p3.fr)
}

Received: 18 July 2012 - Published in Geosci. Instrum. Method. Data Syst. Discuss.: 13 September 2012 Revised: 8 January 2013 - Accepted: 16 January 2013 - Published: 6 February 2013

\begin{abstract}
High-energy (above a few hundred GeV) atmospheric muons are a natural probe for geophysical studies. They can travel through kilometres of rock allowing for a radiography of the density distribution within large structures, like mountains or volcanoes. A collaboration between volcanologists, astroparticle and particle physicists, TOMUVOL was formed in 2009 to study tomographic muon imaging of volcanoes with high-resolution, large-scale tracking detectors. We report on two campaigns of measurements at the flank of the Puy de Dôme using glass resistive plate chambers (GRPCs) developed for particle physics, within the CALICE collaboration.
\end{abstract}

\section{Introduction}

Muons are leptonic elementary particles analogous to their well-known cousins, the electrons, about 200 times heavier with a mass of $105.7 \mathrm{MeV}$. Atmospheric muons are naturally produced, over a wide range of energies and directions, as secondaries of cosmic rays interacting in the earth's atmosphere. Muons are unstable, and they decay solely weakly, $\sim 100 \%$ to electrons, with a rather large proper lifetime in their rest frame, $\tau$, as $c \tau=658.650 \mathrm{~m}$, where $c$ is the vacuum speed of light. Consequently, ultra relativistic highenergy (HE, above a few hundred $\mathrm{GeV}$ ) muons are highly penetrating in matter. They can travel through kilometres of rock before losing all of their energy to the medium or decaying in flight. Indeed, ultra relativistic muons suffer only moderate radiative losses in matter due to their high mass while being close to stable in the terrestrial rest frame. Therefore, atmospheric muons are a relevant probe to perform an absorption measurement through large and dense structures. Early uses included probing Chephren's pyramid (Alvarez et al., 1970) and estimating the rock overburden on mountains (George, 1955). The counting of the number of atmospheric muons surviving through the structure provides a radiographic measurement of the matter distribution along the line of flight of the muons (Nagamine, 2004). If the topography is further known, by repeating the measurement from different locations, tomographic threedimensional models of the matter's density distribution can then be computed (Tanaka et al., 2010). A few percent density contrast over tens of metres spatial resolution is achievable from remote locations, at several kilometres from the target. Hence, muon imaging could locate and characterise in terms of size, depth and shape, magma reservoirs, volcanic conduits and hydrothermal systems, particularly when combined with other techniques used in volcanology, such as seismic, electrical and electromagnetic tomography and satellite interferometry. However, HE atmospheric muons are scarce, with fluxes of $\sim 100 \mathrm{~m}^{-2} \mathrm{deg}^{-1} \mathrm{day}^{-1}$ close to the horizontal (Matsuno et al., 1984). Therefore, accurate measurements require timescales of $\sim 1 \mathrm{month} /(\mathrm{L} / 1 \mathrm{~m})^{2}$, where $L^{2}$ is the area of the muon collection plane. In addition, the 
muons propagation through matter has to be well understood and it is prone to result in model-dependent systematics.

TOMUVOL (Tomographie MUonique des VOLcans) is an interdisciplinary collaboration initiated in 2009, joining particle physicists and volcanologists from three French laboratories: Magmas and Volcanoes Laboratory (LMV) and Laboratory of Corpuscular Physics (LPC), located in ClermontFerrand (Auvergne), and the Lyon Institute of Nuclear Physics (IPNL, Rhône Alpes). It aims to develop muon tomography of volcanoes. The project benefits from a reference site located near Clermont-Ferrand: the Puy de Dôme (alt. 1464 ma.s.l.). The Puy de Dôme is an extinct 11 000yr-old volcanic dome in the Massif Central, south-central France (Miallier et al., 2010; Boivin, 2009). It has a remarkable structure with two domes originating from two subsequent eruptions, which occurred within a short time interval. Its density structure is therefore expected to be complex with large variations. The first phase of the TOMUVOL project started in 2011 and is dedicated to extensive radiographic studies of the Puy de Dôme using glass resistive plate chambers (GRPCs) (Bedjidian et al., 2011), developed for particle physics (CALICE, 2013) as a muon tracker. The results will be compared and combined with gravimetric and electricalresistivity measurements (Portal, 2013). The next step is then the realisation of a precise 3-D tomographic density-map of the Puy de Dôme from multiple observation directions. The second phase, to start in early 2014, is the design, construction and validation of an autonomous and portable radiographic device for volcano tomography (Labazuy, 2013), which can be used for monitoring active volcanoes and for studying their internal structure.

We report here on two preliminary campaigns of measurements of the Puy de Dôme from close to orthogonal directions: Grotte Taillerie and Col de Ceyssat. In Section 2 we briefly review the hardware and software tools used for these measurements. Section 3 is dedicated to a more in-depth discussion of the detection sites and of the experimental setups. Our preliminary results are presented in Sect. 4, and in the concluding section we discuss our prospects.

\section{Tools for muon tomography}

Before discussing in more detail the various hardware and software tools used for the muon tomography, let us first point out that, in order to convert any measured integrated density to an average density, or for a complete tomographic reconstruction, accurate topographical data are needed. Therefore, a dedicated LiDAR survey of the Puy de Dôme and its surroundings was realised in March 2011, thanks to regional funding. An overall precision of better than $10 \mathrm{~cm}$ on a $0.5 \mathrm{~m}$ grid was achieved for the digital elevation model of the area.

\subsection{The GRPC detector used as a muon tracker}

The TOMUVOL muon telescope is made of parallel planes of GRPCs, operated in avalanche mode. This technology allows a high segmentation, in $1 \mathrm{~cm}^{2}$ cells, while being easily scalable up to large areas, $\sim 1 \mathrm{~m}^{2}$. For a typical spacing of $1 \mathrm{~m}$ between the detection planes, it results in $0.5^{\circ}$ angular resolution (evaluated conservatively). In addition, GRPCs are rather cheap, robust, highly efficient $(\sim 0.95 \%)$, with a detection rate up to $100 \mathrm{~Hz}$. They also have a low noise level, less than $1 \mathrm{~Hz} \mathrm{~cm}^{-2}$. A detailed discussion can be found in Laktineh (2013). A single detection plane consists of two parallel thin glass plates kept at a distance of about $1 \mathrm{~mm}$ using tiny ceramic balls as spacers so that a gas mixture $(93 \%$ Forane, $5 \%$ isobutane, $2 \%$ SF6) can circulate between the plates, with a throughput of $\sim 1 \mathrm{Lh}^{-1}$. The outer sides of the glass plates are coated with a thin layer of highly resistive material on which the high voltage (typically $7 \mathrm{kV}$ ) is applied. The high voltage needs to be adapted to the environmental pressure and temperature conditions in order to maintain a constant operating gain. A thin Mylar layer is used as insulation between the anode and the readout: a layer of $1 \mathrm{~cm}^{2}$ copper pads. An ionising charged particle traversing the chamber initiates a fast and highly contained electron avalanche in the gas volume that results in an induced charge in the close-by copper pad(s). The discharge avalanche is limited to a tiny area due to the high resistivity of the glass electrodes and the quenching characteristics of the gas. The chambers are embedded in steel cassettes and are vertically mounted onto a movable aluminium support framework.

The collecting copper pads are assembled on one face of a printed circuit board (PCB) of $50 \times 33.3 \mathrm{~cm}^{2}$. By using six PCBs a total detection area of $1 \mathrm{~m}^{2}$ can be read out. The HARDROC2 readout application-specific integrated circuits, ASICs (Dulucq et al., 2010) each handle $8 \times 8$ pads. In total 48 ASICs are connected on a $1 \mathrm{~m}^{2}$ PCB set, leading to a total of 9142 readout channels per $\mathrm{m}^{2}$. For each pad, ASIC-implemented comparators provide 2-bit digitised amplitude information, with a low and high threshold. In data-taking mode, each ASIC buffers up to 512 successive signals above the low threshold. They are read out through a field-programmable gate array (FPGA) upon receival of an external, periodical trigger signal. The FPGAs manage also the communication with a PC through a USB interface. The data acquisition and monitoring software is based on a cross-platform, distributed framework software (XDAQ, 2013), customised for TOMUVOL.

\subsection{The software tools}

An intensive simulation effort is ongoing within TOMUVOL in order to provide a full simulation of the operating detector as well as to investigate the performances of future detector designs. Furthermore, it is believed that only a detailed simulation can convincingly address the various 
systematic errors that might affect a quantitative density statement. The simulation studies deal with several topics. Detailed air shower simulations are carried out with both the established code within the astroparticle community - CORSIKA (Heck it et al., 1998) - and a more general and flexible one - GEANT4 (Agostinelli et al., 2003) - which is a reference for the particle physics accelerator-community. The latter GEANT 4 code can incorporate a detailed topography and composition of the relief which is of prime importance for close to horizontal muon tracks, and consequently for muon radiography. In addition, these simulations are used to study the background contamination from vertical air showers that are reconstructed as horizontal tracks (Bene, 2013).

In order to estimate the detection efficiency when accounting for dead cells, detector simulations have been carried out with a fast but simplified code. A GEANT4 geometric description of the detector is currently being implemented. It will allow for more detailed efficiency studies by simulating the full detection chain, from the charge signal collection to its digitisation and processing.

Finally, a preliminary simulation scheme has been developed based on a mixed language approach interfacing C++ and Java using Python (Fehr, 2011). The simulation of the atmospheric muon flux relies both on measurements (Matsuno et al., 1984) and theoretical assumptions (Parente et al., 1995). The propagation of muons through rock is handled with the dedicated propagation code MMC (Chirkin and Rhode, 2004), developed for large-scale neutrino detectors. The descriptions of the muon detector and the target geometry are fully customisable. For the geometrical modelling of the Puy de Dôme, the precision topographical data from the LiDAR survey were used.

\section{The radiography measurements}

Two preliminary campaigns of measurements on the Puy de Dôme were carried out in 2011 and 2012. For the first campaign the detector was located at Grotte Taillerie (867 m a.s.1.) from January to July 2011. It was a long-term survey from a location $2 \mathrm{~km}$ east of Puy de Dôme with a $1 / 6 \mathrm{~m}^{2}$ detector. For the second campaign, from February to March 2012, the detector was at Col de Ceyssat (1074 m a.s.l.) $1.2 \mathrm{~km}$ south of Puy de Dôme. It was a shortterm survey from a closer location but with a detector twice as large $\left(1 / 3 \mathrm{~m}^{2}\right)$.

Full simulations have been carried out for the two geometries, assuming a uniform target density of $1.66 \mathrm{~g} \mathrm{~cm}^{-3}$, and a $1 \mathrm{~m}^{2}$ detector. It appears that the Puy de Dôme inner structure should be accessible within a month timescale using a $1 \mathrm{~m}^{2}$ detector, provided that the noise level from fake tracks is controlled at the level of $1 \mathrm{month}^{-1} \mathrm{deg}^{-1} \mathrm{~m}^{-2}$. A radiography of the very base below the Puy de Dôme would however require keeping the background contamination at the level of $1 \mathrm{yr}^{-1} \mathrm{deg}^{-1} \mathrm{~m}^{-2}$.

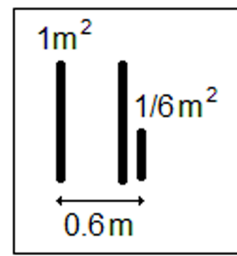

(a)

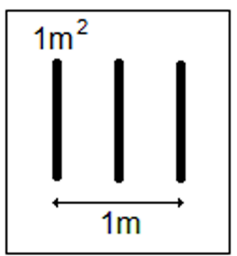

(b)

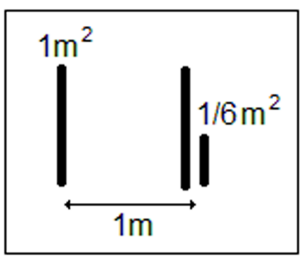

(c)
Fig. 1. Schematic view of the three Grotte Taillerie setups: (a) January-April 2011, (b) April-May 2011, and (c) MayJuly 2011.

\subsection{The Grotte Taillerie campaign}

For the first campaign the detector was deployed in Grotte Taillerie, an underground artificial cave in which it was shielded from vertical atmospheric showers by $60 \mathrm{~cm}$ of concrete. In order to protect the electronics against moisture, due to a high ambient humidity, the detector was placed under a tent of polyethylene foil. Additionally, a dehumidifier ventilated dry air under the tent. The whole setup was controlled remotely from LPC through the long-range Wi-Fi network of the Puy de Dôme summit observatory. This link was also used to transfer the recorded data to a central server. A video camera continuously monitored the installation. Environmental data (i.e. temperature, pressure and humidity) were recorded every $15 \mathrm{~min}$ and are archived in a database.

From January to July 2011, a total of 17 million muon track candidates from the entire sky were recorded with a $91 \%$ duty cycle. Three different detector configurations were actually used, as sketched in Fig. 1, with only a few short interruptions for detector maintenance and systematic tests. The initial setup consisted of two $1 \mathrm{~m}^{2}$ vertical chambers supplemented by a third outer chamber of $1 / 6 \mathrm{~m}^{2}$. The total spacing between the three chambers was $58 \mathrm{~cm}$. Starting 6 April, for three weeks a $1 \mathrm{~m}^{2}$ chamber replaced the small outer chamber and the total spacing increased to $1 \mathrm{~m}$. On 12 May, we put back the small $1 / 6 \mathrm{~m}^{2}$ chamber. A $1 \mathrm{~m}$ distance between the three chambers was however maintained.

The muon detector has been carefully aligned with respect to the Puy de Dôme using GNSS and tachymetric measurements of the surroundings and of the basement thanks to a collaboration with the Ecole Supérieure des Géomètres et Topographes from Le Mans (ESGT). Absolute surface positions are provided by GPS measurements. Reference points in the Taillerie basement were then fixed by tachymetric measurements through the skylights of the basement. Finally the muon detection planes were positioned with respect to these points. The final achieved accuracy on the detector position is better than $5 \mathrm{~mm}$. 

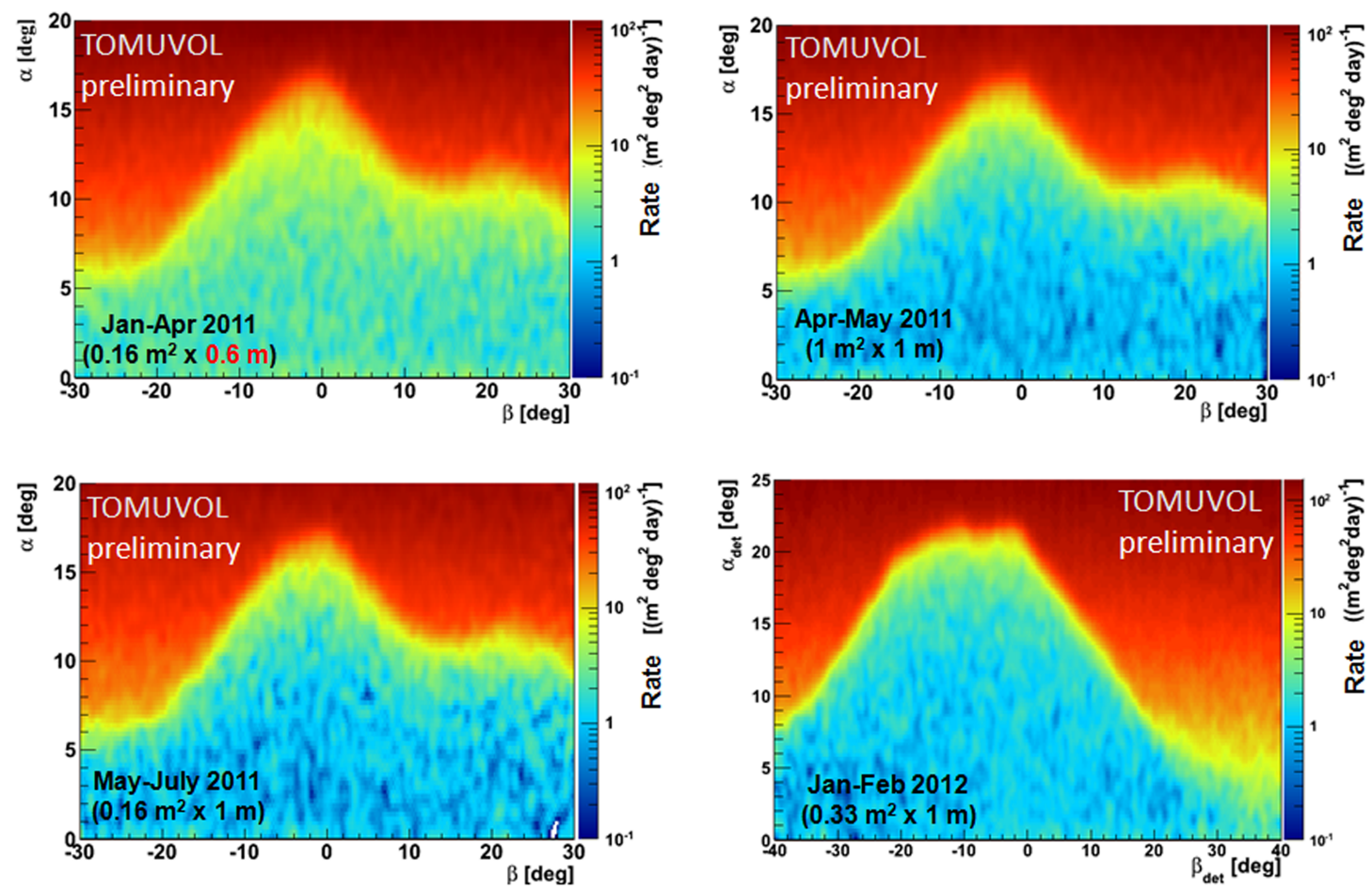

Fig. 2. Shadow cast in the atmospheric muon flux by the Puy de Dôme, as measured with the various setup configurations discussed herein. Note that the detector acceptance has been factored in.

\subsection{The Col de Ceyssat campaign}

In order to get a better view of the Puy de Dôme summit, for the second campaign the detector was installed closer to the volcano at the Gros Manaux Inn. The shielding was only partial from the nearby main building of the inn. In addition, in order to reduce the background rate from fake tracks, a fourth chamber was used. Hence, in total $3 \times 1 \mathrm{~m}^{2}$ vertical chambers and one smaller outer chamber of $1 / 3 \mathrm{~m}^{2}$ were operated. The total spacing was $1 \mathrm{~m}$. We recorded a total of 11 million muon track candidates. As previously the detector was monitored and operated remotely. The positioning, in collaboration with ESGT, was more difficult than in Grotte de la Taillerie due to the detector location within a room with small openings. The estimated accuracy is of $1 \mathrm{~cm}$.

\section{Data processing and preliminary results}

The reconstruction algorithm preselects time coincident hits in the detection planes within a $0.4 \mu$ s window, imposed by the readout electronics. The hit combinations are fitted iteratively to a straight line, accounting for clustering of adjacent hits and track bundles of high multiplicity. The overall $\chi^{2}$ distribution of the track residuals agrees well with the theoretical expectation, assuming Gaussian distributed errors. Overall, a position resolution of $0.4 \mathrm{~cm}$ and an angular resolution better than $0.5^{\circ}$ are achieved for the muon tracks during the first data taking period. The angular resolution is improved by about a factor of 2 for the 1 -m-wide setups. Additionally, the procedure also allows a relative interalignment of the detection planes by letting free the chamber orientation angles in the track fit. For the Grotte Taillerie setup, this allowed to crosscheck the $5 \mathrm{~mm}$ accuracy achieved through GPS and tachometer measurements with the muon tracks themselves.

The shadow cast in the flux of atmospheric muons by the Puy de Dôme, as measured with the various setup configurations, is shown in Fig. 2. For these measurements, the event rates were corrected from the detector geometrical acceptance, dead cells and dead time. Note that there is no correction from individual chamber detection efficiencies yet, which is an additional factor of $\epsilon \sim[0.90 ; 0.95]^{N=3,4}$. In addition, a smoothing algorithm has been applied to the recorded map in order to reduce artificial bands (Moirépatterns) typical of digital images. The observed shape is in good agreement with the actual outline of the volcano. However, it has been found that for the lower part of the Puy de Dôme the recorded track rate decreases by a factor of $\sim 2$ when increasing the chamber spacing from 0.6 to $1 \mathrm{~m}$. This is attributed to background contamination from down-going showers and backscatter close to horizontal tracks. Therefore, with the current data analysis, interpretations are limited to the upper part of the Puy de Dôme, where the muon flux through the rock is above the background. 


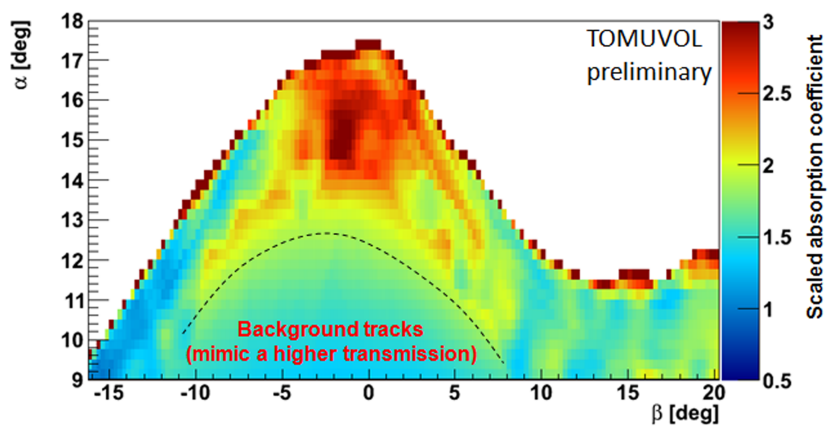

Fig. 3. Map of the scaled transmission through the Puy de Dôme as seen over seven months from the Grotte de la Taillerie with a $\sim 1 / 6 \mathrm{~m}^{2}$ detector.

For illustrative purposes, the full processed data set collected from Grotte de la Taillerie was used in order to compute a tentative scaled transmission coefficient map through the Puy de Dôme. The transmission through the rocks was normalised by the measured open sky flux, and we reported the absorption coefficient corrected for the rock depth for each line of sight as given by the topography (LiDAR measurements). The results are shown in Fig. 3. One can see hints of a structural contrast in the summit region, with apparently a band of higher transmission at the flanks and a region with lower transmission just beneath the summit. At the base of the Puy de Dôme, unfortunately, background tracks mimic a higher transmission.

\section{Conclusion and outlook}

We report on the first extensive muon flux measurements through the Puy de Dôme performed by the ToMUVOL collaboration. The results are very encouraging with $17+11$ million track candidates recorded from two close to orthogonal directions. The detector has proven to work well in an out-of-the-lab environment. The analysis work is ongoing in order to get quantitative results with detailed systematic estimates. In particular, the pollution from background tracks needs to be controlled in order to extend the density image to the lower part of the Puy de Dôme. Yet, it is time for a tentative 3-D tomographic reconstruction of the dome's summit region.

A new detector with a grand total of $1 \mathrm{~m}^{2}$ detection area and a total spacing of $1 \mathrm{~m}$ is to be deployed in 2013. It will integrate four GRPCs with a modular design. It should allow the ultimate radiographic and tomographic imaging of the Puy de Dôme. The design, construction and validation of an autonomous and portable radiographic device for volcano tomography would be the next step.
Acknowledgements. The TOMUVOL collaboration acknowledges support from the University Blaise Pascal of Clermont-Ferrand, CNRS, Région Auvergne, Conseil Général du Puy-de-Dôme. We also thank Jacques Dreher, owner of the Grotte Taillerie du Puy-de-Dôme, who kindly hosted the TOMUVOL detector in Grotte Taillerie for seven months.

Edited by: T. Hurst

\section{References}

Agostinelli, S., Allison, J., Amako, K., Apostolakis, J., Araujo, H., Arce, P., Asai, M., Axen, D., Banerjee, S., Barrand, G., Behner, F., Bellagamba, L., Boudreau, J., Broglia, L., Brunengo, A., Burkhardt, H., Chauvie, S., Chuma, J., Chytracek, R., Cooperman, G., Cosmo, G., Degtyarenko, P., Dell'Acqua, A., Depaola, G., Dietrich, D., Enami, R., Feliciello, A., Ferguson, C., Fesefeldt, H., Folger, G., Foppiano, F., Forti, A., Garelli, S., Giani, S., Giannitrapani, R., Gibin, D., Gómez Cadenas, J. J., González, I., Gracia Abril, G., Greeniaus, G., Greiner, W., Grichine, V., Grossheim, A., Guatelli, S., Gumplinger, P., Hamatsu, R., Hashimoto, K., Hasui, H., Heikkinen, A., Howard, A., Ivanchenko, V., Johnson, A., Jones, F. W., Kallenbach, J., Kanaya, N., Kawabata, M., Kawabata, Y., Kawaguti, M., Kelner, S., Kent, P., Kimura, A., Kodama, T., Kokoulin, R., Kossov, M., Kurashige, H., Lamanna, E., Lampén, T., Lara, V., Lefebure, V., Lei, F., Liendl, M., Lockman, W., Longo, F., Magni, S., Maire, M., Medernach, E., Minamimoto, K., Mora de Freitas, P., Morita, Y., Murakami, K., Nagamatu, M., Nartallo, R., Nieminen, P., Nishimura, T., Ohtsubo, K., Okamura, M., O’Neale, S., Oohata, Y., Paech, K., Perl, J., Pfeiffer, A., Pia, M. G., Ranjard, F., Rybin, A., Sadilov, S., Di Salvo, E., Santin, G., Sasaki, T., Savvas, N., Sawada, Y., Scherer, S., Sei, S., Sirotenko, V., Smith, D., Starkov, N., Stoecker, H., Sulkimo, J., Takahata, M., Tanaka, S., Tcherniaev, E., Safai Tehrani, E., Tropeano, M., Truscott, P., Uno, H., Urban, L., Urban, P., Verderi, M., Walkden, A., Wander, W., Weber, H., Wellisch, J. P., Wenaus, T., Williams, D. C., Wright, D., Yamada, T., Yoshida, H., and Zschiesche, D.: Geant4-a simulation toolkit, Nucl. Inst. Meth. A, 506, 250-303, 2003.

Alvarez, L. W., Anderson, J. A., El Bedwei, F., Burkhard, J., Fakhry, A., Girgis, A., Goneid, A., Hassan, F., Iverson, D., Lynch, G., Miligy, Z., Mohammed-Sharkawi, A. H. M., and Yazolino, L.: Search for Hidden Chambers in the Pyramids, Science, 167, 832839, 1970.

Bedjidian, M., Belkadhi, K., Boudry, V., Combaret, C., Decotigny, D., Cortina Gil, E., de la Taille, C., Dellanegra, R., Gapienko, V. A., Grenier, G., Jauffret, C., Kieffer, R., Fouz, M.-C., Han, R., Laktineh, I., Lumb, N., Manai, K., Mannai, S., Mathez, H., Mirabito, L., Puerta Pelayo, J., Ruan, M., Schirra, F., SeguinMoreau, N., Tromeur, W., Tytgat, M., Vander Donckt, M., and Zaganidis, N.: Performance of Glass Resistive Plate Chambers for a high-granularity semi-digital calorimeter, J. Instrument., 6, P02001, doi:10.1088/1748-0221/6/02/P02001, 2011.

Bene, S.: Atmospheric showers simulation for background estimation for muons tomography, Geosci. Instrum. Method. Data Syst. Discuss., in preparation, 2013.

Boivin, P.: Volcanologie de la Chaîne des Puys, 5th Edn., Parc Naturel Régional des Volcans d'Auvergne, Puy-de-Dôme, France, 196 pp., 2009. 
CALICE: https://twiki.cern.ch/twiki/bin/view/CALICE/WebHome, last access: 5 February 2013.

Chirkin, D. and Rhode, W.: Muon Monte Carlo: A Highprecision tool for muon propagation through matter, arXiv:hepph/0407075, e-Print: hep-ph/0407075 [hep-ph], 37 pp., 2004.

Dulucq, F., de La Taille, C., Martin-Chassard, G., and SeguinMoreau, N.: Nuclear Science Symposium Conference Record (NSS/MIC), IEEE Inst. Elect. Electron. Eng. Publ., 1678-1683, doi:10.1109/NSSMIC.2010.5874060, 2010.

Fehr, F.: Density Imaging of Volcanoes with Atmospheric Muons, ICRC 2011, Proceedings of the 32rd International Cosmic Ray Conference, Beijing, China, 0671, 4 pp., 2011.

George, E. P.: Cosmic rays measure overburden of tunnel, Commonwealth Eng., 455, 455-457, 1955.

Heck, D., Knapp, J., Capdevielle, J. N., Schatz, G., and Thouw, T.: CORSIKA: A Monte Carlo Code to Simulate Extensive Air Showers, Report FZKA 6019, Forschungszentrum Karlsruhe, Karlsruhe, Germany, 90 pp., 1998.

Labazuy, P.: Requirements for a survey system of active volcanoes based on muon radiography, Geosci. Instrum. Method. Data Syst. Discuss., in preparation, 2013.

Laktineh, I.: Development of large GRPC with a very fine segmentation readout electronics, Geosci. Instrum. Method. Data Syst. Discuss., in preparation, 2013.

Matsuno, S., Kajino, F., Kawashima, Y., Kitamura, T., Mitsui, K., Muraki, Y., Ohashi, Y., Okada, A., Suda, T., Minorikawa, Y., Kobayakawa, K., Kamiya, Y., Nakamura, I., and Takahashi, T.: Cosmic-ray muon spectrum up to $20 \mathrm{TeV}$ at $89 \mathrm{deg}$ zenith angle, Phys. Rev. D, 29, 1-23, 1984.
Miallier, D., Boivin, P., Deniel, C., Gourgaud, A., Lanos, P., Sforna, M., and Pilleyre, T.: The ultimate summit eruption of Puy de Dôme volcano (Chaîne des Puys, French Massif Central) about 10,700 years ago, C.R. Geosci., 342, 847-854, doi:10.1016/j.crte.2010.09.004, 2010.

Nagamine, K.: Advanced muon radiography with compact accelerator system, Proc. Jpn. Acad. Ser. B, 80, 179-182, 2004.

Parente, G., Shoup, A., and Yodh, G. B.: Horizontal air showers, atmospheric muons and the cosmic-ray spectrum, Astro. Part. Phys., 3, 17-28, 1995.

Portal, A., Labazuy, P., Lénat, J.-F., Béné, S., Boivin, P., Busato, E., Cârloganu, C., Combaret, C., Dupieux, P., Fehr, F., Gay, P., Laktineh, I., Miallier, D., Mirabito, L., Niess, V., and Vulpescu, B.: Inner structure of the Puy de Dôme volcano: cross-comparison of geophysical models (ERT, gravimetry, muon imaging), Geosci. Instrum. Method. Data Syst., 2, 47-54, doi:10.5194/gi-2-472013, 2013.

Tanaka, H. K. M., Taira, H., Uchida, T., Tanaka, M., Takeo, M., Ohminato, T., Aoki, Y., Nishitama, R., Shoji, D., and Tsuiji, H.: Three-dimensional computational axial tomography scan of a volcano with cosmic ray muon radiography, J. Geophys. Res., 115, B12332, doi:10.1029/2010JB007677, 2010.

XDAQ: https://svnweb.cern.ch/trac/cmsos, last access: 5 February 2013. 